\title{
Two-photon and second harmonic generation microscopy of porcine eye: implications to conductive keratoplasty
}

\author{
Shu-Wen Teng ${ }^{\mathrm{a}}$, Ju-Li Peng ${ }^{\mathrm{b}}$, Huei-Hsing Lin ${ }^{\mathrm{b}}$, Hai-Yin Wu ${ }^{\mathrm{a}}$, Hsin-Yuan Tan ${ }^{\mathrm{c}}$, Wen Lo ${ }^{\mathrm{a}}$, Wei-Chou \\ Lin $^{\mathrm{d}}$, Sung-Jan Lin ${ }^{\mathrm{e}}$, Shiou-Hwa Jee ${ }^{\mathrm{e}, \mathrm{f}}$, Chen-Yuan Dong ${ }^{{ }^{*}}$ \\ ${ }^{a}$ Department of Physics, National Taiwan University, Taipei 106, Taiwan \\ ${ }^{\mathrm{b}}$ Department of Life Science, National Taiwan University, Taipei 106, Taiwan \\ ${ }^{c}$ Department of Ophthalmology, Chang Gung Memorial Hospital, Linko 333, Taiwan \\ ${ }^{\mathrm{d}}$ Department of Pathology, National Taiwan University, Taipei 100, Taiwan \\ ${ }^{e}$ Department of Dermatology, National Taiwan University, Taipei 100, Taiwan \\ ${ }^{\mathrm{f}}$ Department of Dermatology, College of Medicine, National Taiwan University, Taipei 100, Taiwan
}

\begin{abstract}
Two-photon autofluorescence and second harmonic generation (SHG) microscopy are useful imaging techniques for studying tissue components. In this work, we apply this imaging modality to study porcine eye. In particular, we use SHG microscopy to investigate the structural changes to excised porcine corneas and found that this technique is useful for studying its structural changes under thermal treatment.
\end{abstract}

Keywords: two-photon autofluorescence, second harmonic generation, cornea

*Address correspondence to cydong@phys.ntu.edu.tw 


\section{Introduction}

In the decade since the introduction of two-photon fluorescence microscopy, biological non-linear microscopy has developed into a major branch of modern optical microscopy. A number of advantages are associated with this technique. To begin with, the high photon flux necessary for inducing non-linear optical effects from biological specimens limits sample excitation to the focal volume. As a result, imaging using the point-like excitation volume results in images with excellent axial depth discrimination without using confocal apertures. An additional benefit associated with the point-like excitation volume is the reduced photodamage. Furthermore, the near-infrared photons used for sample excitation are scattered and absorbed less by tissue constituents than the UV or visible photons used in one-photon microscopy. Therefore, greater sample imaging depths can be achieved without invasive histological procedures. Finally, the wide spectral separation between the excitation and sample luminescence wavelengths allows a complete detection of the specimen luminescence spectra. ${ }^{1,2}$ Multiphoton microscopy has been successfully applied to a number of fields including transdermal transport, tumor physiology, developmental biology, and neurobiology, ${ }^{3-7}$

In this work, we apply multiphoton microscopy to studying porcine eye. In addition to eye surface imaging, we will also use SHG microscopy to characterize the thermally induced denaturation of cornea collagen.

\section{Materials and methods}

The multiphoton microscope used in this study is similar to one previously described. ${ }^{8}$ In short, a diode-pumped (Millennia, Spectra Physics, Mountain View, CA) femtosecond titanium-sapphire laser (Tsunami, Spectra Physics) was used as the excitation source. The laser is guided toward a modified commercial upright microscope (E800, Nikon, Japan). Prior to entering the microscope, the excitation beam is scanned by an x-y mirror scanning system (Model 6220, Cambridge Technology, Cambridge, MA). At the entrance port of the microscope, a beam expander enlarges the laser beam diameter to ensure overfilling the objective's aperture. The autofluorescence and/or SHG signal generated at the specimen is collected in the epi-illuminated or back-scattered fashion respectively. Single-photon counting PMT's (R7400P, Hamamatsu, Japan) were used to process the signal photons. Porcine eyes were acquired and used for this study.

\section{Results and discussions}

Shown in Fig. 1 is a large area multiphoton scan of the porcine limbus. The structure shown at the right corresponds to the SHG signal generated at the cornea while the fibers shown at the left corresponds to the sclera collagen. In between the cornea and sclera is the autofluroescence from the limbus. From this image, it is clear that multiphoton autofluroescence and SHG signals are efficient at distinguishing cellular structures (autofluorescence) and collagen (SHG). 


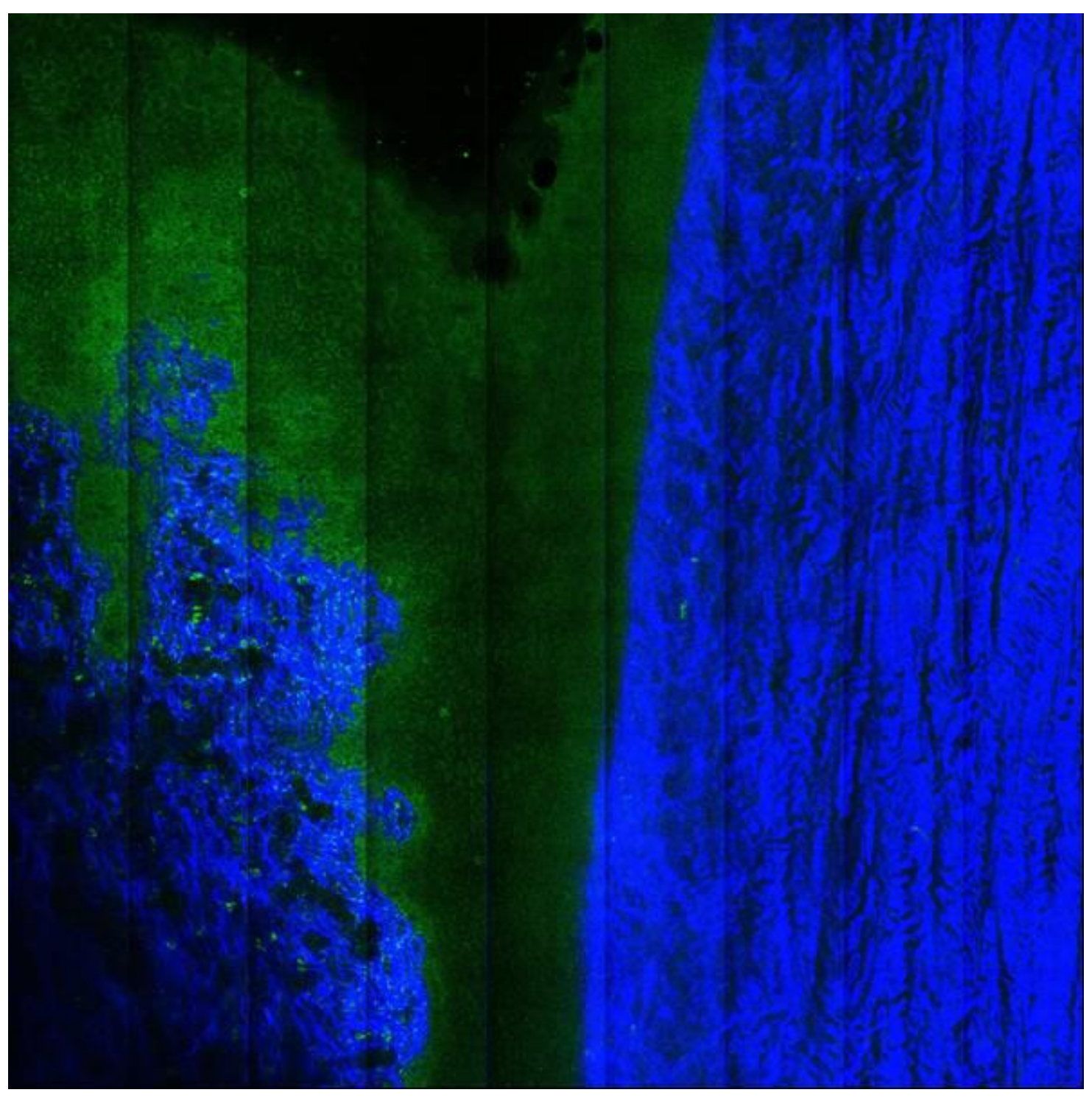

Fig. 1 Large area multiphoton scan of porcine limbus 


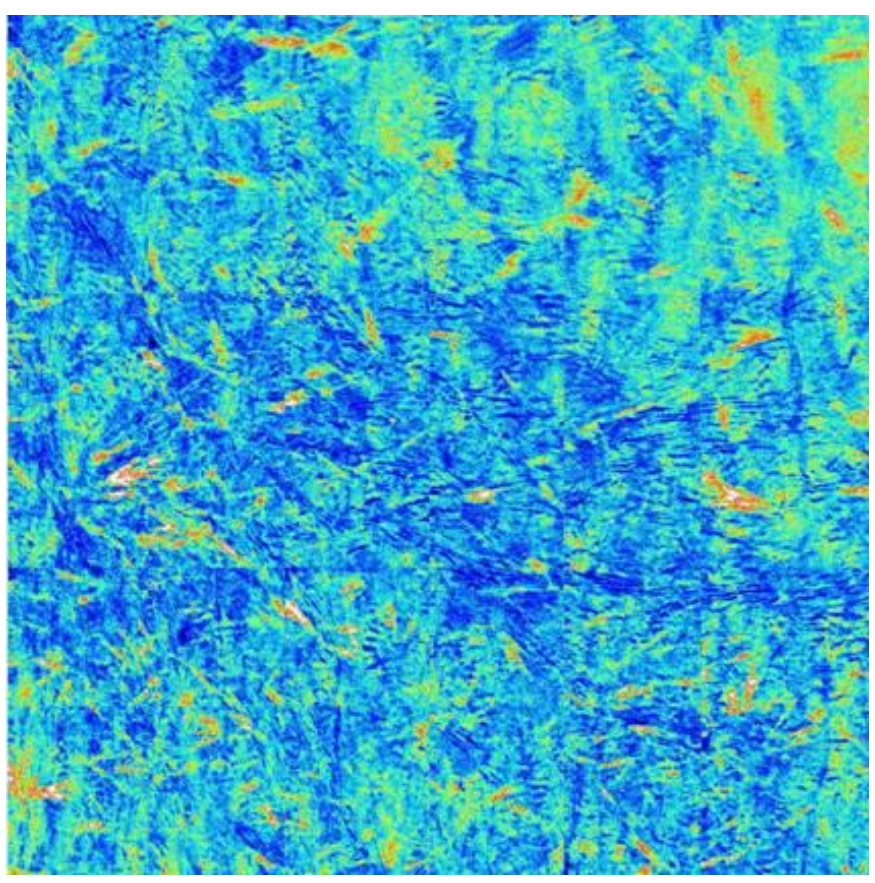

25 degrees Celsius

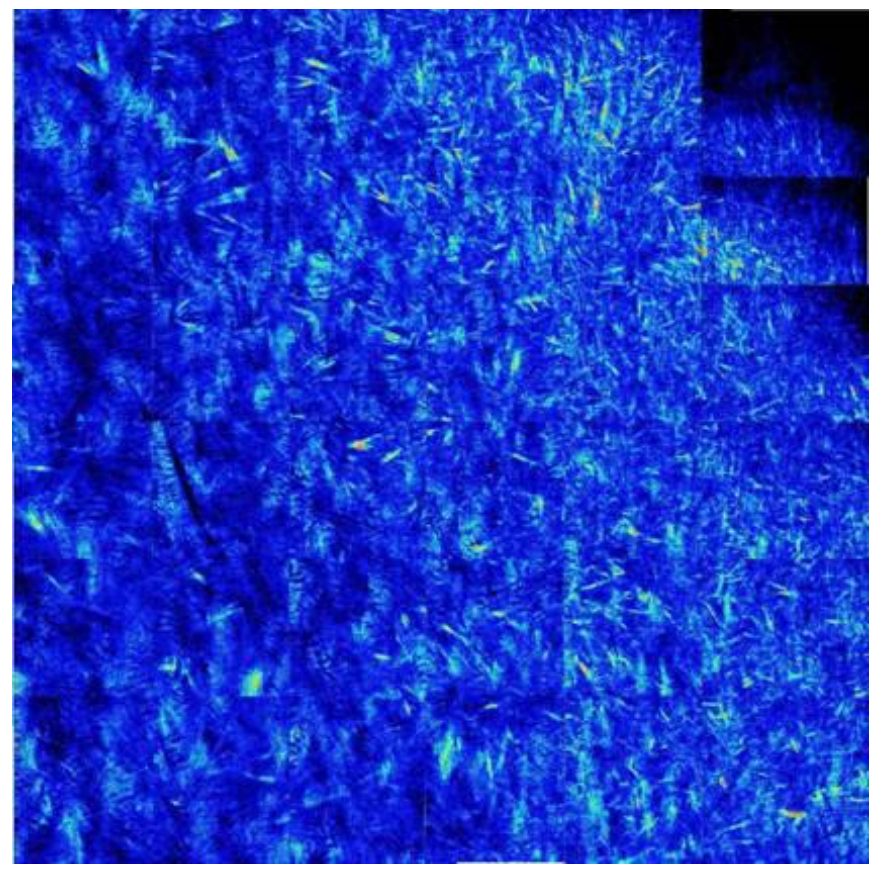

35 degrees Celsius 


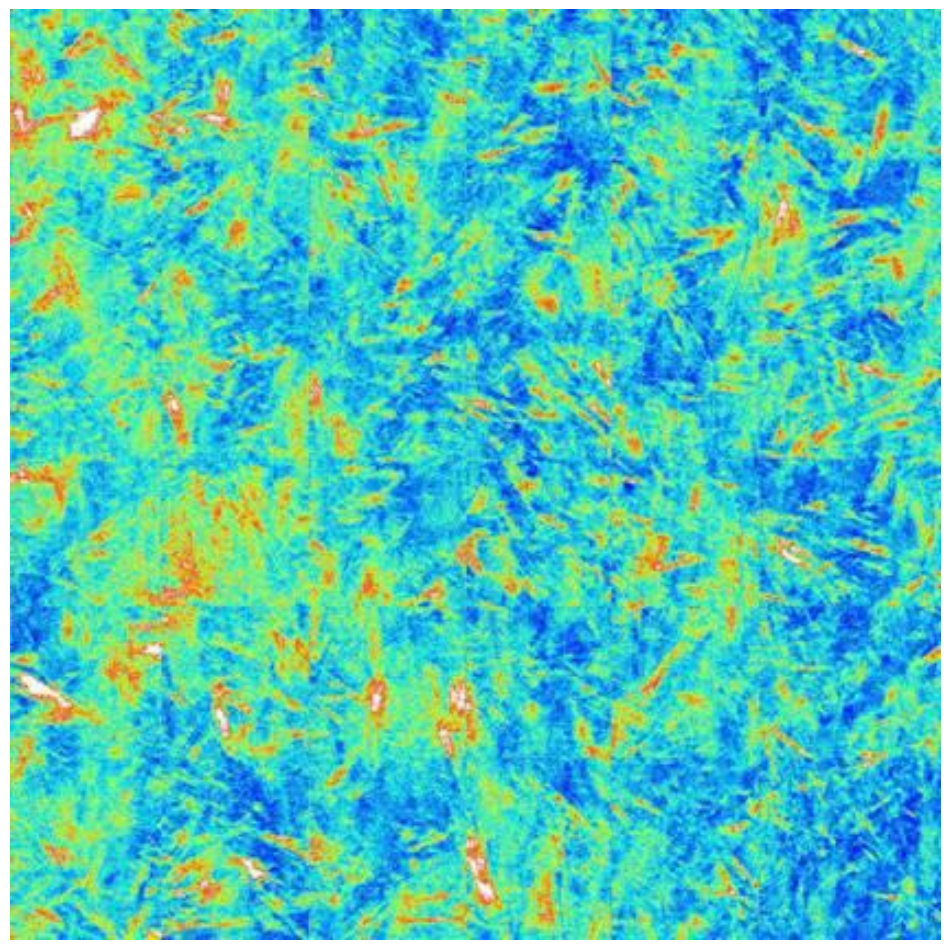

58 degrees Celsius

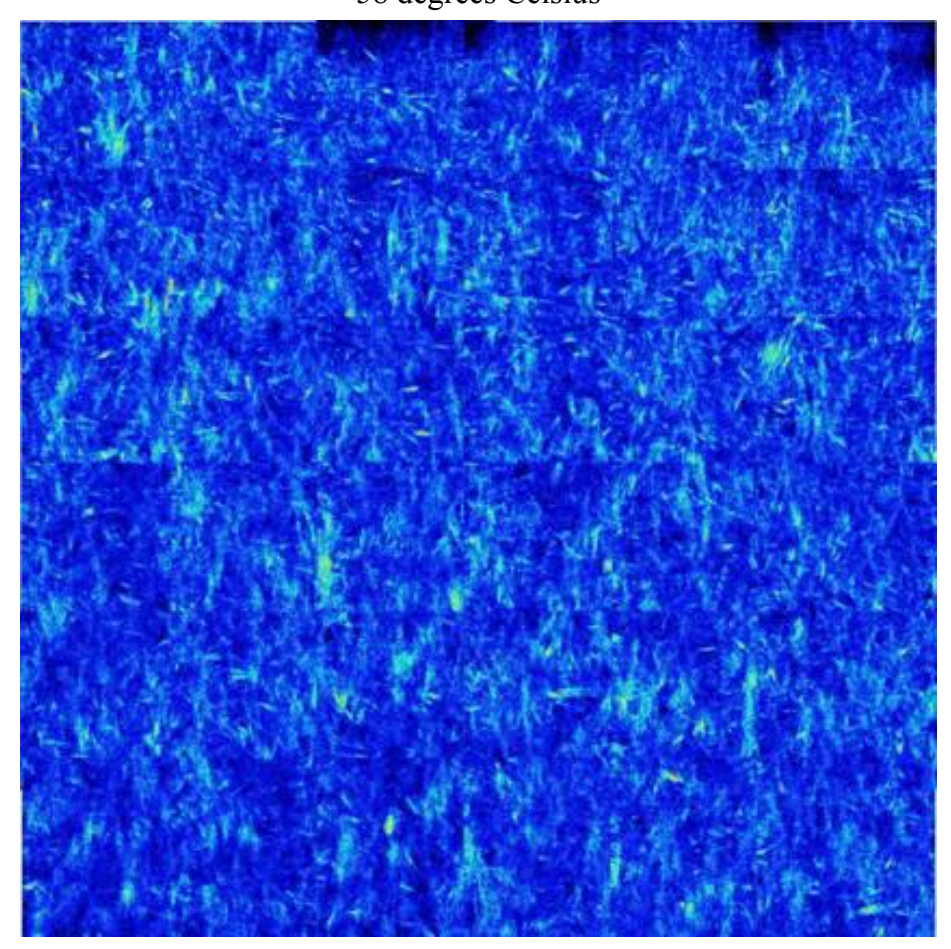

60 degrees Celsius 




70 degrees Celsius

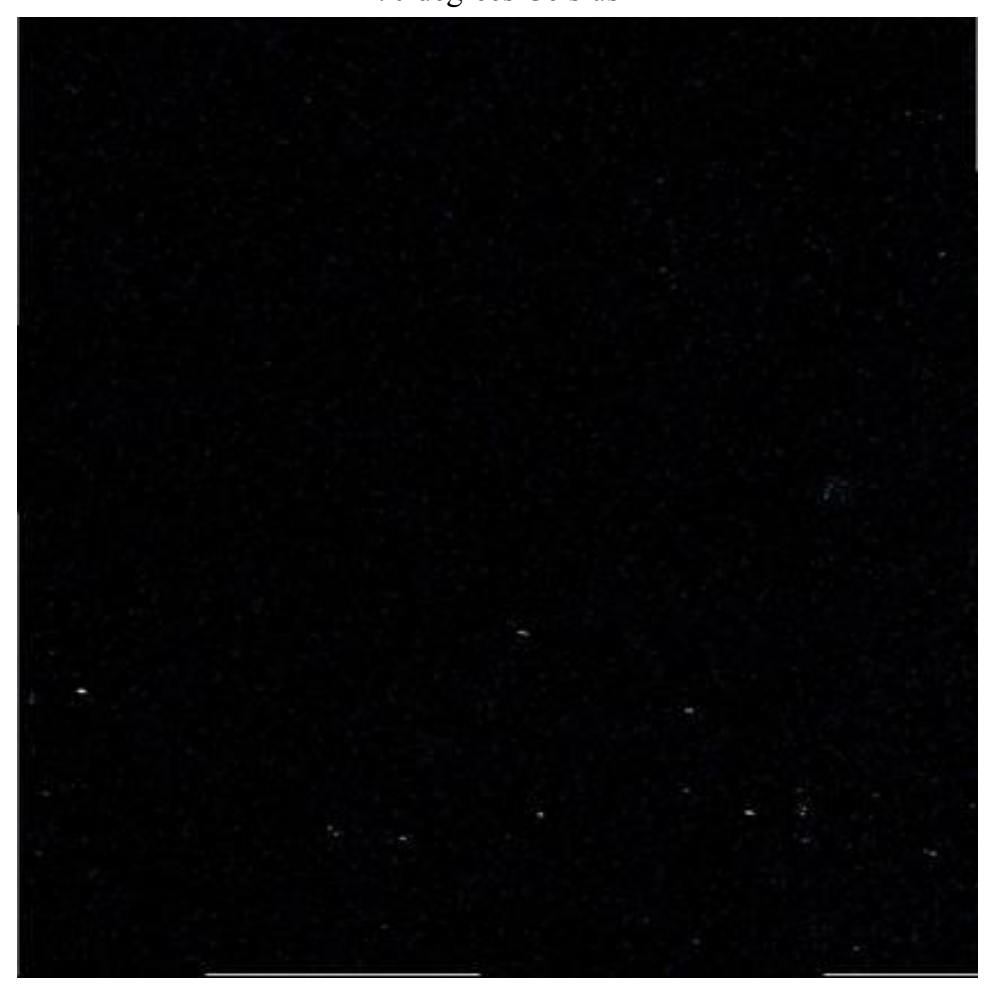

80 degrees Celsius

Fig. 2 SHG images of excised corneas treated at different temperatures 


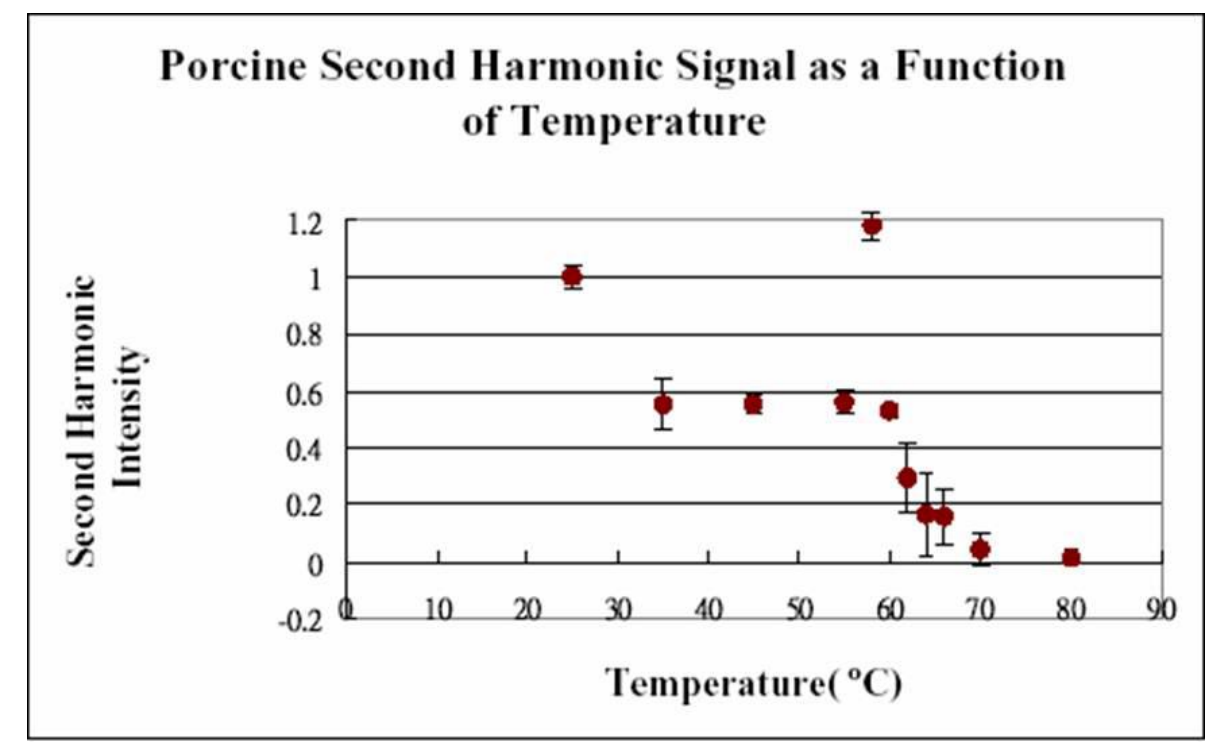

Fig. 3 SHG intensity as a function of temperature of

excised porcine corneas treated at different temperatures

Shown in Fig. 2 are the large area SHG images of excised porcine corneas treated at different temperatures. As is evident from the images, with increasing temperatures, the SHG images have a general tendency of becoming dimmer indicating that the thermal denaturation of cornea collagen results in a decrease in SHG intensity. Shown in Fig. 3 is the SHG intensity as a function of temperature. What is interesting in Fig. 3 is the sudden increase in SHG intensity between 55 and 60 degrees Celsius. The sudden increase can correspond to shrinking of cornea collagen at this temperature range and that results in an increase of SHG intensity.

\section{Conclusions}

In conclusion, we have shown that the combination of multiphoton autofluorescence and SHG imaging is useful in obtaining cellular and collagen structural information of porcine eye. In addition, SHG imaging is capable of identifying thermally induced structural changes to porcine cornea. With additional developments, one can envision multiphoton microscopy to be developed into an effective ophthalmologic imaging technique in vivo.

\section{References}

1. W. Denk, J. H. Strickler, and W. W. Webb, "2-photon laser scanning fluorescence microscopy," Science 248 (4951), 73 (1990).

2. P. T. C. So, C. Y. Dong, B. R. Masters, and K. M. Berland, "Two-photon excitation fluorescence microscopy," 
Annual Review of Biomedical Engineering 2, 399 (2000).

3. B. Yu, C. Y. Dong, P. T. C. So, D. Blankschtein, and R. Langer, "In vitro visualization and quantification of oleic acid induced changes in transdermal transport using two-photon fluorescence microscopy," Journal of Investigative Dermatology 117(1), 16 (2001).

4. Y. Sun, W. Lo, S. J. Lin, S. H. Jee, and C. Y. Dong, "Multiphoton polarization and generalized polarization (GP) microscopy reveals oleic acid induced structural changes in intercellular lipid layers of the skin," Optics Letters, Accepted.

5. E. B. Brown, R. B. Campbell, Y. Tsuzuki, L. Xu, P. Carmeliet, D. Fukumura, and R. K. Jain. "In vivo measurement of gene expression, angiogenesis and physiological function in tumors using multiphoton laser scanning microscopy," Nature Medicine 7(7), 864 (2001).

6. W. Denk, M. Sugimori, R. Llinas. "2 types of calcium response limited to single spines in cerebellar Purkinje-cells," Proceedings of the National Academy of Sciences of the United States of America 92(18), 8279 (1995)

7. J. M. Squirrell, D. L. Wokosin, J. G. White, B. D. Bavister. "Long-term two-photon fluorescence imaging of mammalian embryos without compromising viability,” Nature Biotechnology 17(8), 763 (1999).

8. Y. Sun, J. W. Su, W. Lo, S. J. Lin, S. H. Jee, and C. Y. Dong, "Multiphoton polarization imaging of the stratum corneum and the dermis in ex-vivo human skin," Optics Express 11(25), 3377 (2003).

\section{Acknowledgment}

We acknowledge the support of the NSC 93-3112-B-002-033 (National Science Council, Taiwan) for this project. 